\title{
Adolescent Idiopathic Scoliosis: Males Versus Females
}

\author{
Ebrahim Ameri Mahabadi ${ }^{1}$; Hasan Ghandehari ${ }^{1}$; Seyed Mani Mahdavi ${ }^{1}$; Seyed Hossein \\ Vahid Tari ${ }^{1{ }^{*} ;}$; Ashkan Salimi Sotoudeh ${ }^{1} ;$ Farshad Safdari $^{2}$ \\ ${ }^{1}$ Bone and Joint Reconstruction Research Center, Iran University of Medical Sciences, Tehran, IR Iran \\ ${ }^{2}$ Department of Orthotics and Prosthetics, Bone Joint and Related Tissue Research Center, Shahid Beheshti University of Medical Sciences, Tehran, IR Iran \\ *Corresponding author: Seyed Hossein Vahid Tari, Bone and Joint Reconstruction Research Center, Iran University of Medical Sciences, Tehran, IR Iran. Tel: +98-9128169174, Fax: \\ +98-2133542020, E-mail: tariortho@gmail.com
}

Received: January 22, 2015; Revised: February 13, 2015; Accepted: March 17, 2015

\begin{abstract}
Background: Treatment of adolescent idiopathic scoliosis (AIS) is one of the most challenging problems for spine surgeons. Although it has been previously demonstrated that curve pattern in AIS is different between males and females, there are, however, limited studies specially focused on the differences of AIS characteristics between the two genders.

Objectives: In current study, we compared the demographics and curve patterns in male and female patients with AIS.

Patients and Methods: A total of 68 girls and 17 boys with AIS were included in this cross-sectional study. The magnitude of curvature, thoracic kyphosis, flexibility and pattern of the deformity were measured on x-rays and compared between the two genders.

Results: Although girls were significantly younger (15.3 \pm 2.5 versus $16.7 \pm 2.1$ years; $\mathrm{P}=0.036)$, however, the Risser sign was the same. Most of the males were hypokyphotic (70.6\%), while most of the girls had normal thoracic kyphosis (69.1\%)( $\mathrm{P}=0.001)$. The main scoliotic curve (77.2 \pm 18.2 versus $59.6 \pm 18.6$ degrees) and flexibility ( $25.5 \% \pm 18.2 \%$ versus $41.9 \% \pm 18.6 \%)$ were significantly greater and lower in males, respectively $(\mathrm{P}<0.05)$. The typical AIS pattern was present in $64.7 \%$ of boys and $95.6 \%$ of girls $(\mathrm{P}<0.001)$.

Conclusions: The radiographic characteristics of AIS are substantially different between genders. In male patients, AIS is more severe and rigid compared to females. Also, hypokyphosis and atypical pattern of AIS are common findings in males compared to females.
\end{abstract}

Keywords: Adolescent; Scoliosis; Spine; Kyphosis; Male; Female

\section{Background}

Idiopathic scoliosis is a complicated three dimensional deformity and the most common spinal deformity (1). Adolescent idiopathic scoliosis (AIS) is the most common form of idiopathic scoliosis (over 80\%) with an onset at puberty and female predominance (2-5). The report published by National Scoliosis Foundation in 2006 and previous studies had shown that 1 - 3\% of school children suffer from AIS (6).

It has been demonstrated in previous studies that there are several differences between males and females with AIS. Although males and females are equally affected by mild AIS, however, the severe deformity is more prevalent in girls (2, 7-10). The progression of deformity stops when iliac epiphysis ossifies in girls. However, in boys, usually the curve increases until complete skeletal maturity (Risser V) (11, 12). The literature shows that outcomes of treatment are considerably different between girls and boys (12-18). Furthermore, the sexual differences can influence the extent at which deformity impacts the quality of life and self-image, which is shown by using the Scoliosis Research Society questionnaire (19). To our knowledge, in spite of extensive investigations regarding AIS, there are limited studies comparing the curve pattern and demographics between males and females with AIS (20).

\section{Objectives}

In the current study, we compared the curve pattern and demographic characteristics between Iranian male and female patients.

\section{Patients and Methods}

The study protocol was approved by the ethic board of our institution. Between 2010 and 2012, there were 93 consecutive patients with AIS admitted to Shafa Yahyaian Hospital, Tehran, Iran and participated in the current cross-sectional study. Patients with previous history of surgical treatment, other types of scoliosis, skeletal dysplasia, neuromuscular disorders, endocrine disorders or soft tissue diseases were excluded. Six patients were excluded and the remaining patients underwent physical and radiographic evaluation. Parents were asked to sign the informed consent for participation.

After physical assessment, plain radiography in standing posteroanterior (PA) and lateral views and anterior bending PA view were performed. For lateral x-rays, the patient was asked to bend anteriorly (45 degrees) and completely flex the elbow (21). The magnitude of coronal curve was measured using Cobb's method. The flexibility of the deformity was calculated by the below formula

Copyright (C) 2015, Iran University of Medical Sciences. This is an open-access article distributed under the terms of the Creative Commons Attribution-NonCommercial 4.0 International License (http://creativecommons.org/licenses/by-nc/4.0/) which permits copy and redistribute the material just in noncommercial usages, provided the original work is properly cited. 
and presented as percentage:

(The magnitude of coronal curve in standing PA x-ray the magnitude of coronal curve in anterior bending PA $\mathrm{x}$-ray / the magnitude of coronal curve in standing PA $\mathrm{x}$ ray) $\times 100$.

Also, thoracic kyphosis (TK) was measured using Cobb's method in the sagittal plane. Patients were divided into three groups, based on the magnitude of TK: hypokyphosis (TK $\leq 10$ degrees), normal (TK between 10 - 40 degrees) and hyperkyphosis (TK $\geq 40$ degrees). Magnetic resonance imaging was performed for patients who required surgical intervention and patients with abnormal superficial abdominal reflexes $(22,23)$ and atypical curve pattern (24-26). Two patients were excluded due to the presence of an abnormal neuronal axis, based on MRI.

The location and direction of the coronal curve were recorded (27). On standing PA x-rays, the curve at which the apex passed through the central vertical sacral line was considered as the main scoliotic curve and classified based on the location of the apical vertebra or apical disk space.

In the current study, the curves were classified as below:

- Upper thoracic (curve between T1 and T5 or T6, associated with a right or left $\mathrm{T} 1 \mathrm{tilt}$, or elevation of first or second rib and tilt of the shoulder into the concave side of the upper curve);

- Thoracic (apex between T2 and T11);

- Thoracolumbar (apex at T12, the T12-L1 disc or L1);

- Lumbar (apex at or below L1-L2 disc space).

Regarding the previous classifications for AIS (28) and scoliosis related neuronal axis abnormality $(24,25)$, the main scoliotic curves were divided into two typical and atypical groups. The typical group included:

- The right thoracic curve;

- The right thoracic/left thoracolumbar curve;

- The right thoracic/left lumbar curve;

- The thoracolumbar curve;

- The lumbar curve.

Also, the atypical curve patterns included:

- The left thoracic curve;

- The left thoracic/right lumbar curve;

- The left thoracic/right thoracolumbar curve;

- The right and left double thoracic curve;

- The right long thoracic (King IV) curve;

- The right and left triple curve;

- The quadruple curve.

Between typical curves, if the below patterns were present, the deformity was considered as AIS with typical feature (based on the location of the apex and typical level)
$(25,26)$ :

- The right thoracic curve (T5 - 6 to T11 - 12, apex T7 - 8);

- The right thoracic/left thoracolumbar curve [King 1 (right thoracic: T4 - 6 toT11, apex T8; left thoracolumbar T11 to L3 - 4, apex L2), KingII (right thoracic: T5 to T11, apex T9; left thoracolumbar T11 - L4, apex L2)];

- The right thoracic/left lumbar curve (right thoracic T3 T5 to T10, apexT4 - T5; left lumbar T10 - L3, apex L1);

- The thoracolumbar curve (T9 - T10 to L3, apex T12 - L1);

- The lumbar curve (T12 - L4, apex L2).

Other cases of typical scoliosis that had no typical feature were considered as AIS with atypical feature.

The statistical analysis was performed with the statistical software SPSS version 15 (SPPS Inc., Chicago, IL, USA). Quantitative data were compared between males and females using independent samples t-test or Mann-Whitney U test. The Quantitative data were compared utilizing chi-square test or Fisher's exact test. A P $<0.05$ was considered significant.

\section{Results}

Eighty five patients aged $15.6 \pm 2.5$ years (range: $12-20$ years) completed the study. There were 17 males (20\%) and 68 females (80\%). The male patients were significantly older $(15.3 \pm 2.5$ versus $16.7 \pm 2.1 ; \mathrm{P}=0.036)$. However, the Risser signs of the two groups did not differ significantly $(\mathrm{P}=0.929)$. Most of the males were hypokyphotic $(70.6 \%)$, while most of the girls had normal TK $(69.1 \%)(P=0.001)$. Table 1 compares the magnitude of main scoliotic curve, the magnitude of the curve in anterior bending and the flexibility of the curve between girls and boys, showing that deformity and flexibility are significantly more severe and situated lower in boys, respectively.

Most of the patients of two groups had typical pattern of AIS (64.7\% in males, versus $95.6 \%$ in females) and the difference was statistically significant $(\mathrm{P}<0.001)$. Tables 2 and 3 represent the frequency of typical and atypical pattern of AIS in the two groups. As shown, most of the patients had right thoracic curve (63.6\% of males and 53.8\% of females) followed by right thoracic/left lumbar pattern in the girls group (23.1\%). In term of type of deformity, the difference between the two groups was not significant ( $P$ $>0.05$ ). Between patients with typical deformity, there were only 11 patients with atypical feature, including nine girls and two boys $(\mathrm{P}=0.656)$. The frequency of typical features between patients with typical pattern of AIS is presented in Table 4, revealing no significant difference between girls and boys $(\mathrm{P}=0.729)$.

Table 1. Comparing the Curve Magnitude in Standing and Anterior Bending Postures and the Flexibility of the Curve Between Males and Females

\begin{tabular}{lccc}
\hline Group & Males $(\mathbf{N}=\mathbf{1 7})$ & Females $(\mathbf{N}=\mathbf{6 8})$ & P Value \\
\hline Curve magnitude in standing posture (degree) & $77.2 \pm 18.2(42-104)$ & $59.6 \pm 18.6(32-120)$ & 0.002 \\
Curve magnitude in anterior bending posture (degree) & $58.4 \pm 20.7(18-90)$ & $36 \pm 19(8-100)$ & $<0.001$ \\
Flexibility (\%) & $25.5 \pm 18.2(2.2-64)$ & $41.9 \pm 18.6(4-81)$ & 0.002 \\
\hline
\end{tabular}


Ameri Mahabadi E et al.

Table 2. The Frequency of Typical Patterns in Males and Females

\begin{tabular}{lccc}
\hline Type of Typical Deformity & Males $(\mathbf{N}=\mathbf{1 1})$ & Females $(\mathbf{N}=\mathbf{6 5})$ & Total \\
\hline Right thoracic & 7 & 35 & 42 \\
Right thoracic/left thoracolumbar & 1 & 3 & 4 \\
Right thoracic/left lumbar & 1 & 15 & 16 \\
Thoracolumbar & 2 & 8 & 10 \\
Lumbar & 0 & 4 & 4 \\
Total & 11 & 65 & 76 \\
\hline
\end{tabular}

Table 3. The Frequency of Atypical Patterns in Males and Females

\begin{tabular}{lccc}
\hline Type of Atypical Deformity & Males $(\mathbf{N}=\mathbf{6})$ & Females $(\mathbf{N}=\mathbf{3})$ & Total \\
\hline Left thoracic & 1 & 1 & 2 \\
\hline Left thoracic/ right lumbar & 0 & 1 & 1 \\
\hline Left thoracic/ right thoracolumbar & 0 & 0 & 0 \\
\hline Right and left double thoracic & 3 & 1 & 4 \\
Right long thoracic (King IV) & 0 & 0 & 0 \\
Right long triple curve pattern & 1 & 1 & 2 \\
\hline Right long quadruple curve pattern & 0 & 0 & 0 \\
Total & 6 & 3 & 9 \\
\hline
\end{tabular}

Table 4. The Frequency of Features of Typical Patterns in Males and Females

\begin{tabular}{lccc}
\hline Type of Feature & Males $(\mathbf{N}=\mathbf{1 1})$ & Females $(\mathbf{N}=\mathbf{6 5})$ & Total \\
\hline Right thoracic & 6 & 32 & 38 \\
\hline Left thoracic/ right lumbar & 1 & 5 & 6 \\
\hline Right thoracic/ left thoracolumbar (King I) & 0 & 9 & 9 \\
\hline Right thoracic/ left thoracolumbar (King II) & 0 & 1 & 1 \\
Right thoracic/ left lumbar & 2 & 7 & 9 \\
Thoracolumbar & 0 & 3 & 3 \\
\hline Lumbar & 0 & 0 & 0 \\
Atypical feature & 2 & 8 & 10 \\
Total & 11 & 65 & 76 \\
\hline
\end{tabular}

\section{Discussion}

The AIS is one of the most challenging deformities in adolescence and can be associated with several physical, psychological and financial problems for the patient, his/ her family and healthcare system. The treatment is always long lasting and complicated and, in spite of all of the efforts and advances, it is not successful in several cases. It seems that one of the more important causes of treatment failure is incomplete knowledge of the nature of AIS and etiologic factors affecting the deformity and its prognosis. The prognosis of AIS is affected by several factors, such as morphology of the curve, severity, age of onset, skeletal growth and rate of deformity progression. The AIS may resolve spontaneously, with growth, or may progress severely and even be associated with risk of mortality $(1,15)$. The progressive deformity may cause several body prominences, asymmetric waistline, uneven shoulders, injured self-image, pain, spinal degenerative changes, limitations in activities of daily living and, in severe cases, disturbed pulmonary function (29-32).

In many cases, surgical intervention is necessary to improve self-image and pulmonary function and to prevent the progression of deformity, which has been reported to be associated with favorable results (33). The rate of correction had been reported at $60-80 \%$, utilizing modern instruments (34-36).

Several authors demonstrated a number of differences between girls and boys with AIS, in terms of pattern of the deformity, which should be considered in treatment. However, to our knowledge, although there are many studies regarding AIS, only a limited number of them 
compared the characteristics of the deformity between males and females.

Recently, Suh et al. performed an epidemiologic survey in Korean adolescents and found that AIS is more prevalent in girls (4.65\% vs. 1.97\%). Also, they demonstrated that the curve pattern and severity of deformity are different in male and female patients. Only $3.91 \%$ of boys vs. $11.28 \%$ of girls had a curve between 20 - 29 degrees (37). In a similar study in China, Wang et al. (20) compared the curve pattern and other radiographic variables between 359 males and 999 females with AIS $\geq 20$ degrees. They found that atypical pattern is more prevalent in males $(19.8 \%$ versus $8.9 \%$ ). They also demonstrated that the type of deformity is different between genders; however, the main thoracic curve was the most important curve type in males and females. In patients with severe typical AIS, including a major thoracic curve, the magnitude of thoracic scoliotic curve and TK were greater and the deformity was more rigid in males (20). Our findings confirm the previous studies. We found that the two genders are significantly different in terms of radiographic parameters. Although the males were significantly older than the females, however, the skeletal age was the same, based on the Risser sign. Most of the boys were hypokyphotic (70\%), while most of the girls had normal kyphosis (about 70\%). The main scoliotic curve was more severe and rigid in male patients. The typical pattern of AIS was found in $95.6 \%$ of girls, while atypical pattern was found in a considerable fraction of the boys (35.3\%). However, the differences were not significant. The most prevalent typical pattern for both males and females was right thoracic pattern (63.6\% in males and $53.8 \%$ in females), followed by right thoracic/left lumbar in girls (23.1\%). Similarly, the most frequent typical feature in both genders was right thoracic feature. In contrast to our findings, in a retrospective study on Iranian patients, Ameri et al. observed that the pattern and severity of the deformity were the same between the two genders. However, they found that the curve was more rigid in males (18).

Although not investigated in the current study, several previous authors have found different outcomes of surgical or nonsurgical treatment between male and female patients (12-18). For example, Yrjonen et al. found significantly more curve progression with non-operative treatment in male patients (31.4\% vs. $21.6 \%$ ) and explained poor compliance with brace wear, as one of the potential reasons (13). However, others did not confirm these findings. Ameri et al. demonstrated that preoperative sexual differences did not affect the radiological outcomes of surgical treatment of AIS (18). Also, Marks et al. found similar results in their study (17).

With regard to several controversies in findings of studies, it seems necessary to compare the characteristics of the AIS and its course in males and females, in future prospective studies. There are several unsolved questions regarding the AIS and such studies can help to explain several of these problems. In a review study, Raggio
(7) explained that gender differences in spine growth, morphology, stiffness, curve pattern and hormones, in combination with genetic factors, may contribute to the phenotype of the scoliosis. Also, he believed that these factors may affect the curve progression and response to treatment. Raggio explained that sexual dimorphism can impact diagnosis, treatment and outcomes (7).

Our study was limited by the number of male patients, which can bias the findings. Also, we investigated the patients of only one hospital and, for judging the differences between males and females with AIS, it is necessary to perform more extensive studies.

In conclusion, based on the findings of the current study, the radiographic characteristics of AIS differ significantly between genders. The AIS is more severe and rigid in male patients. Hypokyphosis is present in most of the boys, while the normal kyphosis is found in most of the girls. Also, in contrast to the typical pattern of the deformity found in near all of the female patients, a substantial percentage of the male patients developed an atypical pattern of the deformity.

\section{Authors' Contributions}

Ebrahim Ameri Mahabadi; Hasan Ghandehari; Seyed Mani Mahdavi; Seyed Hossein Vahid Tari; Ashkan Salimi Sotoudeh; Farshad Safdari. Study concept, design, drafting the manuscript, and critical revision of the manuscript: Ebrahim Ameri Mahabadi; Hasan Ghandehari; Seyed Mani Mahdavi; Seyed Hossein Vahid Tari. Analysis and interpretation of data: Ashkan Salimi Sotoudeh; Farshad Safdari. Study supervision: Hasan Ghandehari.

\section{Financial Disclosure}

The authors did not receive any funding or grants in support of their research or preparation for this work. No funding source played a role in the study.

\section{References}

1. Gorman KF, Julien C, Moreau A. The genetic epidemiology of idiopathic scoliosis. Eur Spine J. 2012;21(10):1905-19.

2. Weinstein SL, Dolan LA, Cheng JCY, Danielsson A, Morcuende JA. Adolescent idiopathic scoliosis. The Lancet. 2008; 371(9623):1527-37.

3. Wang WJ, Yeung HY, Chu WC, Tang NL, Lee KM, Qiu Y, et al. Top theories for the etiopathogenesis of adolescent idiopathic scoliosis. J Pediatr Orthop. 2011;31(1 Suppl):S14-27.

4. Weiss HR, Goodall D. The treatment of adolescent idiopathic scoliosis (AIS) according to present evidence. A systematic review. Eur J Phys Rehabil Med. 2008;44(2):177-93.

5. Riseborough EJ, Wynne-Davies R. A genetic survey of idiopathic scoliosis in Boston, Massachusetts. J Bone Joint Surg Am. 1973;55(5):974-82.

6. Willner S, Uden A. A Prospective Prevalence Study of Scoliosis in Southern Sweden. Acta Orthop .1982;53(2):233-7.

7. Raggio CL. Sexual dimorphism in adolescent idiopathic scoliosis. Orthop Clin North Am. 2006;37(4):555-8.

8. Luk KD, Lee CF, Cheung KM, Cheng JC, Ng BK, Lam TP, et al. Clinical effectiveness of school screening for adolescent idiopathic scoliosis: a large population-based retrospective cohort study. Spine (Phila Pa 1976). 2010;35(17):1607-14. 
9. Ueno M, Takaso M, Nakazawa T, Imura T, Saito W, Shintani R, et al. A 5-year epidemiological study on the prevalence rate of idiopathic scoliosis in Tokyo: school screening of more than 250,000 children. J Orthop Sci. 2011;16(1):1-6.

10. Suh PB, MacEwen GD. Idiopathic Scoliosis in Males A Natural History Study. Spine. 1988;13(10):1091-4.

11. Karol L. Male adolescent idiopathic scoliosis. West $\mathrm{J}$ Med. 1993;159(4):482-3.

12. Karol LA. Effectiveness of Bracing in Male Patients With Idiopathic Scoliosis. Spine. 2001;26(18):2001-5.

13. Yrjonen T, Ylikoski M, Schlenzka D, Poussa M. Results of brace treatment of adolescent idiopathic scoliosis in boys compared with girls: a retrospective study of 102 patients treated with the Boston brace. Eur Spine J. 2007;16(3):393-7.

14. Siu King Cheung C, Tak Keung Lee W, Kit Tse Y, Ping Tang S, Man Lee K, Guo X, et al. Abnormal peri-pubertal anthropometric measurements and growth pattern in adolescent idiopathic scoliosis: a study of 598 patients. Spine (Phila Pa 1976). 2003;28(18):2152-7.

15. Sucato DJ, Hedequist D, Karol LA. Operative correction of adolescent idiopathic scoliosis in male patients. A radiographic and functional outcome comparison with female patients. $J$ Bone Joint Surg Am. 2004;86-A(9):2005-14.

16. Helenius I, Remes V, Yrjonen T, Ylikoski M, Schlenzka D, Helenius $\mathrm{M}$, et al. Does gender affect outcome of surgery in adolescent idiopathic scoliosis? Spine (Phila Pa 1976). 2005;30(4):462-7.

17. Marks M, Petcharaporn M, Betz RR, Clements D, Lenke L, Newton PO. Outcomes of surgical treatment in male versus female adolescent idiopathic scoliosis patients. Spine (Phila Pa 1976). 2007;32(5):544-9.

18. Ameri E, Behtash H, Mobini B, Omidi-Kashani F, Momeni B. Radiographic outcome of surgical treatment of adolescent idiopathic scoliosis in males versus females. Scoliosis. 2008;3:12.

19. Roberts DW, Savage JW, Schwartz DG, Carreon LY, Sucato DJ, Sanders JO, et al. Male-female differences in Scoliosis Research Society-30 scores in adolescent idiopathic scoliosis. Spine (Phila Pa 1976). 2011;36(1):E53-9.

20. Wang W, Zhu Z, Zhu F, Sun C, Wang Z, Sun X, et al. Different curve pattern and other radiographical characteristics in male and female patients with adolescent idiopathic scoliosis. Spine (Phila Pa 1976). 2012;37(18):1586-92.

21. Janssen MM, Drevelle X, Humbert L, Skalli W, Castelein RM. Differences in male and female spino-pelvic alignment in asymptomatic young adults: a three-dimensional analysis using upright low-dose digital biplanar X-rays. Spine (Phila Pa 1976). 2009;34(23):E826-32.

22. Saifuddin A, Tucker S, Taylor BA, Noordeen MH, Lehovsky J. Prevalence and clinical significance of superficial abdominal reflex abnormalities in idiopathic scoliosis. Eur Spine J. 2005;14(9):849-53.
23. Nakahara D, Yonezawa I, Kobanawa K, Sakoda J, Nojiri H, Kamano S, et al. Magnetic Resonance Imaging Evaluation of Patients With Idiopathic Scoliosis. Spine. 2011;36(7):E482-5.

24. Spiegel DA, Flynn JM, Stasikelis PJ, Dormans JP, Drummond DS, Gabriel KR, et al. Scoliotic curve patterns in patients with Chiari I malformation and/or syringomyelia. Spine (Phila Pa 1976). 2003;28(18):2139-46.

25. Qiu Y, Zhu Z, Wang B, Yu Y, Qian B, Zhu F. Radiological presentations in relation to curve severity in scoliosis associated with syringomyelia. J Pediatr Orthop. 2008;28(1):128-33.

26. Coonrad RW, Murrell GAC, Motley G, Lytle E, Hey LA. A Logical Coronal Pattern Classification of 2,000 Consecutive Idiopathic Scoliosis Cases Based on the Scoliosis Research Society-Defined Apical Vertebra. Spine. 1998;23(12):1380-91.

27. Bitan FD, Veliskakis KP, Campbell BC. Differences in the Risser grading systems in the United States and France. Clin Orthop Relat Res. 2005(436):190-5.

28. Nachemson AL, Peterson LE. Effectiveness of treatment with a brace in girls who have adolescent idiopathic scoliosis. A prospective, controlled study based on data from the Brace Study of the Scoliosis Research Society. J Bone Joint Surg Am. 1995;77(6):815-22.

29. Trobisch P, Suess O, Schwab F. Idiopathic scoliosis. Dtsch Arztebl Int. 2010;107(49):875-83.

30. Ameri E, Behtash H, Mobini B, Bouzari B, Tari V. Patient satisfaction after scoliosis surgery. Med J Islam Repub Iran. 2008; 21(4):177-84

31. Carter OD, Haynes SG. Prevalence Rates for Scoliosis in US Adults: Results from the First National Health and Nutrition Examination Survey. Int J Epidemiol. 1987;16(4):537-44.

32. Schwab F, Dubey A, Gamez L, El Fegoun AB, Hwang K, Pagala M, et al. Adult Scoliosis: Prevalence, SF-36, and Nutritional Parameters in an Elderly Volunteer Population. Spine. 2005;30(9):1082-5.

33. Tolo VT. Surgical treatment of adolescent idiopathic scoliosis. Semin Spine Surg Scoliosis. 1991;3:220-9.

34. Suk SI, Kim JH, Kim SS, Lee JJ, Han YT. Thoracoplasty in thoracic adolescent idiopathic scoliosis. Spine (Phila Pa 1976). 2008;33(10):1061-7.

35. Bullmann V, Halm HF, Niemeyer T, Hackenberg L, Liljenqvist U. Dual-rod correction and instrumentation of idiopathic scoliosis with the Halm-Zielke instrumentation. Spine (Phila Pa 1976). 2003;28(12):1306-13.

36. Lonner BS, Auerbach JD, Estreicher M, Milby AH, Kean KE. Videoassisted thoracoscopic spinal fusion compared with posterior spinal fusion with thoracic pedicle screws for thoracic adolescent idiopathic scoliosis. JBone Joint Surg Am. 2009;91(2):398-408.

37. Suh SW, Modi HN, Yang JH, Hong JY. Idiopathic scoliosis in Korean schoolchildren: a prospective screening study of over 1 million children. Eur Spine J. 2011;20(7):1087-94. 